\title{
Locally produced mucosal IgG in chickens immunized with conventional vaccines for Newcastle disease virus
}

\author{
S. Chimeno Zoth ${ }^{1,2}$, E. Gómez ${ }^{1,2}$, E. Carrillo ${ }^{1,2}$ and A. Berinstein ${ }^{1,2}$ \\ ${ }^{1}$ Instituto de Biotecnología, Centro de Investigaciones en Ciencias Veterinarias y Agronómicas, Instituto \\ Nacional de Tecnología Agropecuaria, Buenos Aires, Argentina \\ ${ }^{2}$ Consejo Nacional de Investigaciones Científicas y Técnicas (CONICET), Buenos Aires, Argentina
}

Correspondence to: A. Berinstein, Instituto de Biotecnología, Centro de Investigaciones en Ciencias Veterinarias y Agronómicas, Instituto Nacional de Tecnología Agropecuaria, Cc 25, B1712WAA, Castelar, Buenos Aires, Argentina

Fax: +54-11-4621-0199. E-mail: aberinstein@cnia.inta.gov.ar

\begin{abstract}
Newcastle disease virus (NDV) is the causative agent of an economically important disease, which affects all species of birds worldwide. Current vaccination programs for NDV include the use of either low-virulent live-virus vaccines or inactivated vaccines to induce protective immunity while producing minimal adverse effects in birds. In order to further characterize the immune response elicited by live virus and inactivated NDV conventional vaccines in chickens, we evaluated the presence of specific antibodies in different secretions and in tissue culture supernatants of immunized birds. To this end, we analyzed all the samples by ELISA, using an indirect assay set up in the laboratory. Specific anti-NDV IgG antibodies were detected in tracheal and cloacal swabs and tracheal and intestinal washes of immunized animals. We also found specific anti-NDV IgG antibodies in tracheal and intestinal tissue culture supernatants, indicating that the IgG found in swabs and washes was not transudated from serum or, at least, was not all transudated from serum. Knowledge about the mechanisms involved in the immune response of chickens to different NDV vaccines should increase our understanding of the mucosal response against the virus and, eventually, provide new useful information for the development and evaluation of synthetic vaccines.
\end{abstract}

Key words: Newcastle disease virus vaccines; Systemic immune response; Mucosal immune response

Research supported by grant PICTO 08-12919 from Agencia Nacional de Promoción Científica y Tecnológica, Argentina and PE AEGR 3423 from Instituto Nacional de Tecnología Agropecuaria, Argentina.

Received July 25, 2007. Accepted February 18, 2008

\section{Introduction}

Newcastle disease virus (NDV), a member of the genus Avulavirus within the Paramyxoviridae family (1), causes an economically important disease in all species of birds worldwide (2). The disease can vary from clinically inapparent to highly virulent forms depending on the virus strain and host species (2). Highly virulent NDV infection is a disease notifiable to the World Organization for Animal Health (3), because it is the cause of sanitary obstacles for the trade of avian products among countries (4).

Current vaccination programs for NDV include the use of either low-virulent live-virus vaccines or inactivated vaccines to induce protective immunity while producing minimal adverse effects in birds. Inactivated oil-emulsion vaccines are manufactured for use by individual bird injection. On the other hand, low-virulent infectious virus may be ingested or inhaled and is the basis for mass application of live-virus vaccines in drinking water or by large droplet aerosol. Birds may also be inoculated with eye drops containing vaccines based on attenuated viruses.

Vaccination using nonvirulent NDV strains protects susceptible birds against disease, producing an antibody response either locally, systemically or both. Several stud- 
ies have reported that the mucosal application of attenuated live virus induces both systemic and local immunity, while parenteral immunization with inactivated vaccines generally induces systemic immunity (5-10).

The aim of the present study was to further characterize the immune responses elicited by live and inactivated NDV vaccines in chickens by evaluating the specific antibodies present in serum samples, in different secretions and in culture supernatants of tissue from immunized birds.

Knowledge about the mechanisms involved in the immune response of chickens to different NDV vaccines should increase our understanding of the mucosal response against the virus and eventually supply new information useful for the development and evaluation of synthetic vaccines.

\section{Material and Methods}

\section{Animals}

White Leghorn-specific pathogen-free embryonated chicken eggs were obtained from Laboratorios Inmuner S.A.I.C. (Argentina) and incubated in an automatic incubator until hatching. Feed and water were provided ad libitum.

\section{Vaccines}

Commercially available inactivated oil emulsion (Laboratorios Inmuner S.A.I.C.) and intra-ocular (ND clone 30 from Nobilis ${ }^{\circledR}$ ) NDV vaccines were used. Both vaccines contained lentogenic NDV as antigen. Recommended vaccine doses were used $(0.25 \mathrm{~mL} /$ dose $)$.

\section{Experimental design}

Fifteen-day-old chickens were randomly divided into three groups and housed in individual cages with free access to water and food. Animals were immunized on days 0 and 14 . Eight chickens were given inactivated oil emulsion vaccines intramuscularly, 8 chickens were given live vaccine in eyedrops, and 8 chickens remained unvaccinated.

Blood samples were collected weekly after the first immunization from a wing vein, allowed to clot for $4 \mathrm{~h}$ and centrifuged. The sera obtained were kept at $-20^{\circ} \mathrm{C}$ until used. Twenty-one days after the first vaccination, tracheal and cloacal swabs were obtained and placed in phosphate-buffered saline (PBS) supplemented with protease inhibitors (P8340, Sigma-Aldrich, St. Louis, MO, USA). Animals were then sacrificed, and tracheas and intestines were dissected. The organs were immersed in $10 \mathrm{~mL}$ PBS, an aliquot of PBS was taken (wash samples) and then tracheal and intestine tissues were cut into small pieces and washed intensively with PBS containing $2 \times 10^{5} \mathrm{U} / \mathrm{L}$ penicillin, $13 \%$ streptomycin, and $10 \%$ gentamicin (tissue samples). Tissue samples were then incubated at $41^{\circ} \mathrm{C}$ in $5 \% \mathrm{CO}_{2}$ in 24-well plates containing RPMI 1640 medium supplemented with $10 \%$ fetal calf serum and the antibiotics described above. After three days, supernatant solutions were collected (tissue culture supernatant samples) and stored at $-20^{\circ} \mathrm{C}$ until used.

\section{Enzyme-linked immunosorbent assay}

An indirect enzyme-linked immunosorbent assay (ELISA) was used to measure chicken IgG against NDV. Briefly, 96-well plates (Maxisorp NUNC ${ }^{\text {TM }}$, USA) were coated with purified La Sota virus antigen (11) in $0.1 \mathrm{M}$ bicarbonate buffer, $\mathrm{pH} 9.6$, overnight, at $4^{\circ} \mathrm{C}$. After blocking with $4 \%$ skim milk in PBS-T-ENS (0.05\% Tween 20, 0.5\% equine normal serum), the plates were incubated with the different samples and with goat anti-chicken IgG antibodies coupled to horseradish peroxidase (Bethyl Laboratories, Inc., USA). Fifty microliters of a solution containing $600 \mu \mathrm{L} 1.7 \%(\mathrm{w} / \mathrm{v})$ ABTS [2,2'-azino-bis (3-ethylbenthiozoline-6-sulfonic acid) diammonium salt] (ICN Biomedicals, $\mathrm{OH}, \mathrm{USA}), 12 \mu \mathrm{L} 30 \%(\mathrm{v} / \mathrm{v}) \mathrm{H}_{2} \mathrm{O}_{2}$ and $25 \mathrm{~mL}$ citric acid buffer, $\mathrm{pH} 5$, was added to each well as substrate. The absorbance of the samples was measured at $405 \mathrm{~nm}$. Samples were analyzed individually in duplicate and the result is reported as the mean value of both wells.

\section{Hemagglutination inhibition test}

The hemagglutination inhibition $(\mathrm{HI})$ assay was performed by a conventional microtiter method as described by King (12) using four hemagglutinating units of NDV La Sota strain. The titers were reported as the $\log _{10}$ of the inverse of the highest sample dilution showing complete $\mathrm{HI}$. Samples were analyzed individually in duplicate.

\section{Viral neutralization assay}

Pools of serum samples were inactivated for $30 \mathrm{~min}$ at $56^{\circ} \mathrm{C}$ and serially diluted 4-fold in 199 medium. All dilutions were incubated in the presence of 100 plaque formation units of NDV Beaudette strain, for $1 \mathrm{~h}$ at room temperature and for $30 \mathrm{~min}$ at $4^{\circ} \mathrm{C}$. Then, each mix was added to a primary chicken embryo fibroblast cell culture seeded in 6 well plates and the plates were incubated for $1 \mathrm{~h}$ at $37^{\circ} \mathrm{C}$. Finally, cells were overlaid with minimum essential medium containing $2 \%$ fetal bovine serum, 25 mM HEPES, $10 \%$ antibiotic-antimycotic agents, and $0.8 \%$ agarose low gelling temperature. The plates were incubated at $37^{\circ} \mathrm{C}$ in $5 \%$ $\mathrm{CO}_{2}$ for 4 days. The cells were then fixed with $10 \%$ formol in PBS and stained with crystal violet for plaque observa- 
tion. Neutralizing titers are reported as the $\log _{10}$ of the inverse of the sample dilution that showed $50 \%$ of plaque formation inhibition.

\section{Statistical analysis}

The data obtained in the ELISA tests and $\mathrm{HI}$ assays were analyzed by the Student $t$-test and means were compared by the Bonferroni test using the Statistixs 7.0, version$^{\odot}$ 1985, 2000 Analytical Software (USA).

\section{Results}

Serum antibody response induced by vaccination

The kinetics of IgG production was essentially similar for both inactivated and low-virulent virus vaccines (Figure 1A). However, IgG induced by low-virulent virus was detectable at day 7 post-vaccination, while inactivated NDV vaccine induced a detectable response from day 14 postvaccination. Both vaccines induced a significant production of IgG in chickens by day 14 post-vaccination, with the highest titer detected on day 21 .

The $\mathrm{HI}$ antibody responses of vaccinated animals at different times post-vaccination are shown in Figure 1B. An antibody response was detected in all inoculated birds, whereas none was detected in control chickens (Figure 1B). The HI titer was first detected at day 7 post-vaccination in birds vaccinated with the low-virulent virus vaccine, and reached a plateau at day 14 post-vaccination. In contrast, after immunization with the inactivated vaccine, $\mathrm{HI}$ titers were not detected until 14 days post-vaccination and reached the highest titer on day 21. Again, both inactivated and low-virulent virus vaccines induced the significant production of antibodies after day 14 postvaccination.

Figure $1 \mathrm{C}$ shows the results of the sero-neutralization assay. Low-virulent virus vaccine induced sero-neutralization activity detectable from day 7 post-vaccination, while chickens vaccinated with the inactivated NDV vaccine showed a solid response from day 14 post-vaccination. Again, the highest titer recorded on day 21 was detected in the group of birds receiving the inactivated NDV vaccine. No sero-neutralization response was detected in untreated chickens (Figure 1C).

The results of the $\mathrm{HI}$ test in general agreed, in terms of kinetics, with the results of ELISA and of the sero-neutralization assay.

Antibody response in swabs and washes

IgG antibody levels in cloacal and tracheal swabs were measured by ELISA 21 days post-vaccination (Figure 2A). A significant antibody response was detected in tracheal
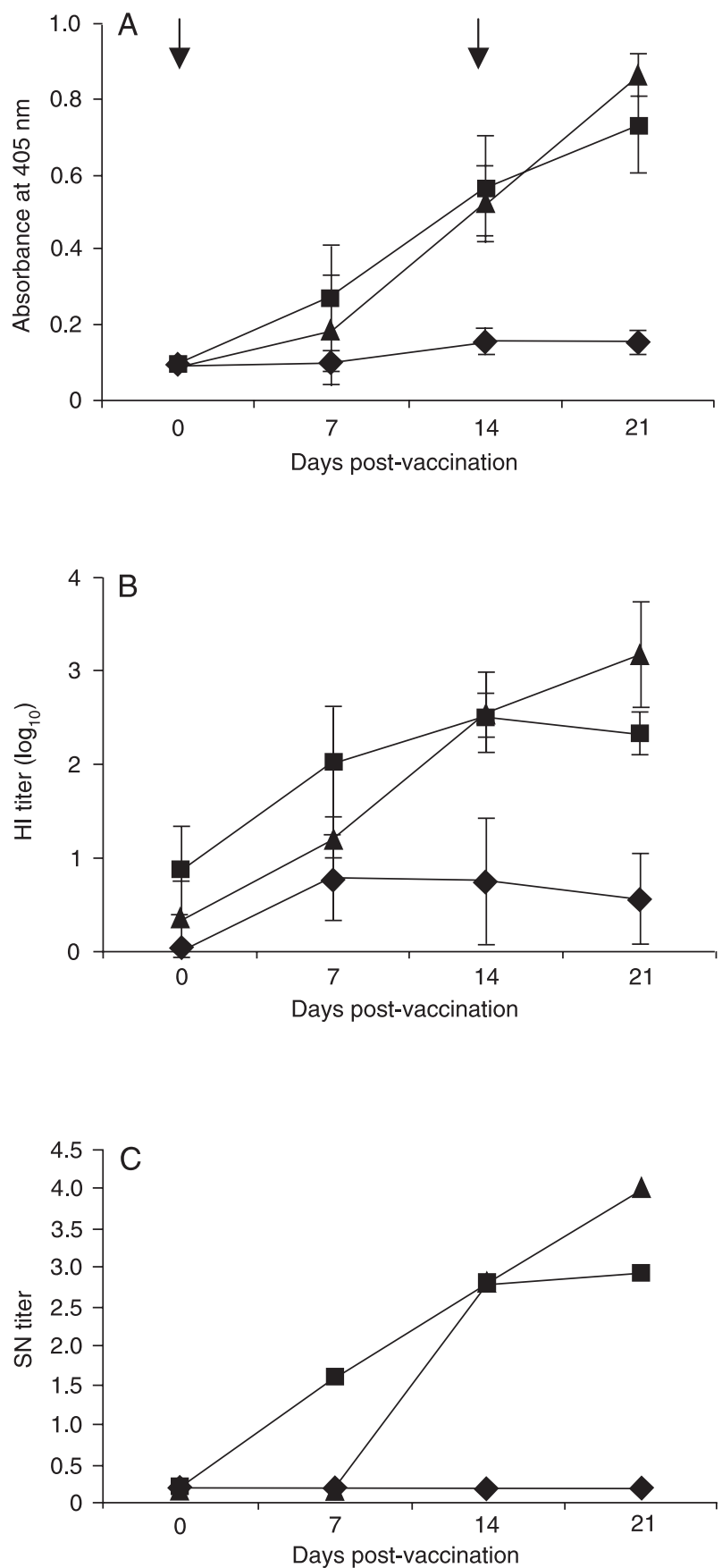

Figure 1. Humoral immune response to Newcastle disease virus in serum samples. Chickens were vaccinated at days 0 and 14 (arrows) with low-virulent virus (squares) or inactivated (triangles) vaccines. Unvaccinated animals are indicated by lozenges. Serum samples were collected weekly and analyzed by $A$, ELISA; $B$, hemagglutination inhibition $(\mathrm{HI})$ test, and $C$, sero-neutralization (SN) assay. Each result is the mean \pm SD for eight samples. The sero-neutralization assay was performed using pooled samples. 

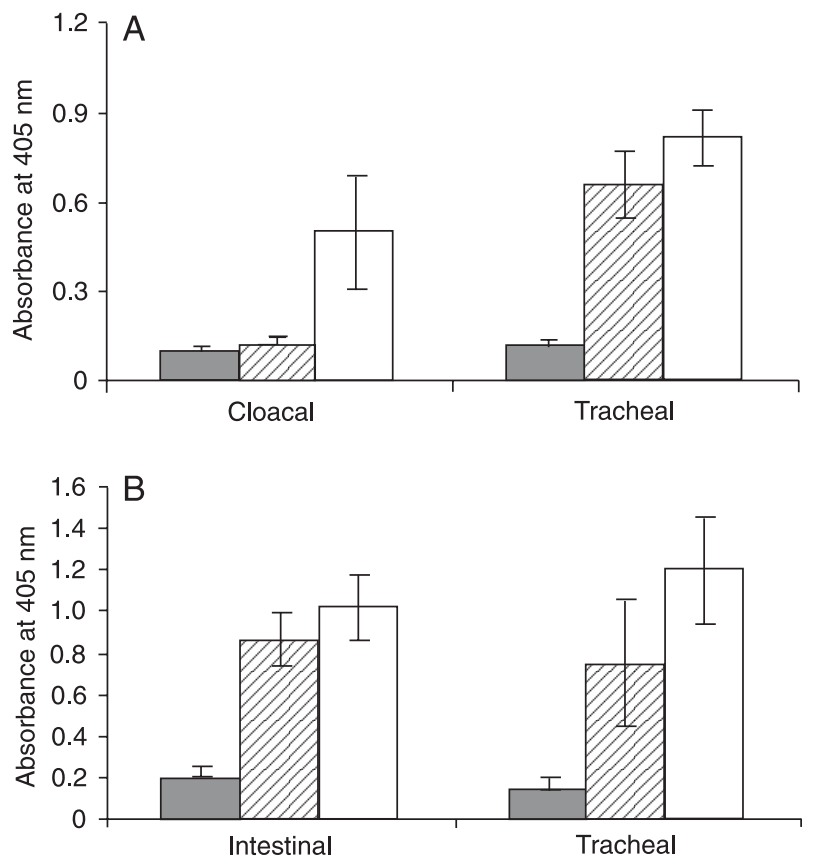

Figure 2. IgG response to Newcastle disease virus (NDV) in cloacal and tracheal swabs and wash samples. Chickens were vaccinated at days 0 and 14 with low-virulent virus (striped columns) or inactivated (open columns) vaccines. Unvaccinated animals are indicated by closed columns. Twenty-one days after the first vaccination swab samples were collected and analyzed by ELISA (A). Each result represents the mean \pm SD for eight samples. After swab sampling, chickens were sacrificed and anti-NDV antibodies were detected in intestinal and tracheal washes by ELISA (B). Each result is the mean \pm SD for eight samples.

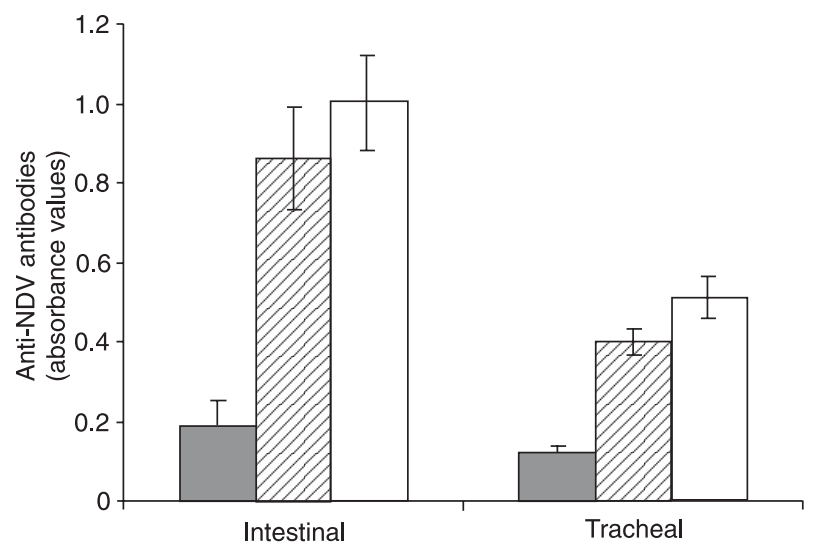

Figure 3. IgG response to Newcastle disease virus (NVD) in tissue culture supernatants. Chickens were vaccinated at days 0 and 14 with low-virulent virus (striped columns) or inactivated (open columns) vaccine. Unvaccinated animals are indicated by closed columns. On day 21 chickens were sacrificed and antiNDV antibodies were detected in intestinal and tracheal fragment culture supernatants by ELISA. Each result is the mean \pm SD for eight samples. swabs from both groups of immunized animals, with a higher titer being found in animals vaccinated with inactivated NDV $(P<0.05)$. Interestingly, when cloacal swabs were analyzed, a significant production of anti-NDV IgG was detected only in birds immunized with the inactivated NDV vaccine and no response was observed in birds receiving the low-virulent virus vaccine (Figure $2 \mathrm{~A}, \mathrm{P}<$ 0.05). No response was detected in samples from untreated animals.

NDV-specific antibodies were also determined in tracheal and intestinal washes. On day 21 all chickens were sacrificed, tracheal and intestinal washes were collected and NDV-specific IgG antibodies were measured by ELISA (Figure 2B). High $(P<0.05)$ IgG antibody levels were observed in both tracheal and intestinal washes from both groups (Figure $2 \mathrm{~B}$ ). Untreated birds showed no response at all (Figure 2B).

\section{Antibody response in tissue culture supernatants}

Tissue fragment cultures were examined to determine if NDV-specific antibodies observed in tracheal and intestinal washes were originated in situ. No antibody response was observed in culture supernatants obtained at time 0 (data not shown), allowing a consistent analysis of the in situ antibody production. Solid anti-NDV-specific IgG antibody responses were detected in the supernatants of 3day cultured small intestine fragments of chickens immunized with both vaccines (Figure $3, \mathrm{P}<0.05$ compared to the negative control). Anti-NDV IgG antibodies were also present in tracheal tissue supernatants (Figure 3).

These results suggest the presence of anti-NDV-specific IgG-secreting plasma cells in the intestinal and tracheal tissues at the time of sample collection from immunized chickens.

\section{Discussion}

In the present investigation, we studied the response elicited by low-virulent live-virus and inactivated vaccines against NDV in chickens, evaluating the presence of IgG in different fluids and trying to identify IgG production sites.

As previously reported by others, we found anti-NDV IgG antibodies in sera of vaccinated animals, regardless of the vaccine used for immunization. Both live and inactivated viruses elicited a strong antibody response in serum, and antibodies were detected by ELISA, HI test or seroneutralization assays (Figure 1). In agreement with the results obtained by Al-Garib and co-workers (13), animals receiving live-virus vaccine were the first to show a specific immune response, while those receiving the inactivated vaccine showed higher levels of antibodies at 21 days 
post-vaccination. We did not determine whether antibodies contributed to protection, but it is well known that both vaccines confer total protection against the disease $(14,15)$.

IgG was also present in tracheal swabs and tracheal washes from immunized animals (Figure 2). The fact that we found IgG in tracheal tissue culture supernatants (Figure 3) indicates that the IgG found in tracheal swabs and washes was not transudated from serum or, at least, was not all transudated from serum, since our results suggest that there are stimulated IgG-secreting plasma cells in the trachea. These results agree with the early suggestion that Igs other than IgA may have a role in the protection of tracheal mucosa against NDV (16). Similarly, we found anti-NDV IgG antibodies in intestinal tissue culture supernatants, indicating that there are specific stimulated plasma cells in the chicken's gut, and, again, that the IgG found in intestinal washes was not all transudated from serum.

The small differences observed in the response to live and inactivated viruses may be due to different antigen handling. Replicating agents which are applied locally, still reach the circulation and thus could stimulate lymphoid cells in systemic lymphoid organs. However, no antibodies were found in cloacal swabs from animals receiving the live vaccine (Figure 2), and in all samples analyzed the level of antibodies of this group of animals was lower than the one found in the samples of chickens vaccinated with the inactivated vaccine. This fact could be due to the nature of the strain present in the live vaccine (La Sota NDV) and its ability to spread through the animal's body. Different strains of NDV are classified as virulent (including velogenic and mesogenic viruses) and non-virulent (including lentogenic and asymptomatic viruses) pathotypes (17). The strain-specific difference in virulence is determined, among other mechanisms, by structural variations of the fusion glycoprotein $(F)$ and the cleavage of the precursor glycoprotein $\left(F_{0}\right)$ into $F_{1}$ and $F_{2}$ subunits $(18,19)$. This cleavage is mediated by host cell proteases (18). The presence of multibasic amino acids at the $\mathrm{F}$ protein cleavage site and the presence of phenylalanine at position 117

\section{References}

1. Mayo MA. A summary of taxonomic changes recently approved by ICTV. Arch Virol 2002; 147: 1655-1663.

2. Alexander DJ. Newcastle disease and avian paramixovirus infections. In: Calnek BW, Barnes HJ, Beard CW, McDougald LR, Saif YM (Editors), Diseases of poultry. 10th edn. Ames: lowa State University Press; 1997. p 541-569.

3. Office International des Epizooties. Terrestrial animal health code. Office International des Epizooties www.oie.int; 2003.

4. Office International des Epizooties. Manual for animal dis- are typical for virulent strains, which are able to infect a wide range of tissues, whereas strains that have single basic residues are non-virulent and are able to infect some tissues only. La Sota NDV is a lentogenic virus and this could explain the results obtained here, i.e., in all samples analyzed, the level of antibodies of animals vaccinated with the live vaccine was lower than the one found in the samples of chickens vaccinated with the inactivated vaccine.

The La Sota NDV strain was also present in the inactivated vaccine, but the route of inoculation and the immunization doses might have helped the induction of antibody production in different tissues, even when the production of antibodies in secreta generally is thought to be stimulated by live antigens.

We were unable to detect anti-NDV IgA antibodies by ELISA in the samples (data not shown). This might be due to competition caused by large amounts of $\lg G$ antibodies present in them; we are working on the assay trying to solve this matter. Interestingly, Al Garib and co-workers (13) found a lower IgA response in chickens inoculated with the La Sota strain than in animals inoculated with a more virulent strain, and almost no IgA response in chickens inoculated with inactivated NDV.

The results obtained in the present study indicate that both live-attenuated and inactivated vaccines induce an anti-NDV antibody response both in serum and intestinal and tracheal secretions and that both vaccines stimulate the production of $\lg$ by plasma cells present in both tissues.

\section{Acknowledgments}

The authors are grateful to Dr. Osvaldo Zabal (Instituto de Virología, Centro de Investigaciones en Ciencias Veterinarias y Agronómicas, Instituto Nacional de Tecnología Agropecuaria, Buenos Aires, Argentina) for collaboration during animal sampling and to Teresa Morán, Jorge Lorenzo and Silvio Díaz for excellent technical assistance. ease reporting to the OIE. Paris: World Organization for Animal Health; 1996.

5. Aitken ID, Parry SH, Powell JR, Survashe BD. Local immunity in Newcastle disease: some recent experiments. Dev Biol Stand 1976; 33: 302-308.

6. Ewert D. The local immune response of the chicken to Newcastle disease virus. Diss Abstr Int 1977; 7: 3343-3344.

7. Jayawardane GW, Spradbrow PB. Mucosal immunity in chickens vaccinated with the V4 strain of Newcastle dis- 
ease virus. Vet Microbiol 1995; 46: 69-77.

8. Levy R, Spira G, Zakay-Rones Z. Newcastle disease virus pathogenesis in the respiratory tract of local or systemic immunized chickens. Avian Dis 1975; 19: 700-706.

9. Parry SH, Aitken ID. Immunoglobulin A in the respiratory tract of the chicken following exposure to Newcastle disease virus. Vet Rec 1973; 93: 258-260.

10. Zakay-Rones Z, Levy R. Immunologic response of chicks to inactivated Newcastle disease virus. Avian Dis 1973; 17: 450-452.

11. Berinstein A, Vazquez-Rovere C, Asurmendi S, Gomez E, Zanetti $\mathrm{F}$, Zabal O, et al. Mucosal and systemic immunization elicited by Newcastle disease virus (NDV) transgenic plants as antigens. Vaccine 2005; 23: 5583-5589.

12. King DJ. Avian paramyxovirus type 1 from pigeons: isolate characterization and pathogenicity after chicken or embryo passage of selected isolates. Avian Dis 1996; 40: 707-714.

13. Al-Garib SO, Gielkens AL, Gruys DE, Hartog L, Koch G. Immunoglobulin class distribution of systemic and mucosal antibody responses to Newcastle disease in chickens. Avian Dis 2003; 47: 32-40.

14. Czifra G, Mészáros J, Horváth E, Moving V, Engström BE.
Detection of NDV-specific antibodies and the level of protection provided by a single vaccination in young chickens. Avian Pathol 1998; 27: 562-565.

15. Gallili GE, Ben-Nathan D. Newcastle disease vaccines. Biotechnol Adv 1998; 16: 343-366.

16. Ewert DL, Eidson CS. Effect of bursectomy and depletion of immunoglobulin $\mathrm{A}$ on antibody production and resistance to respiratory challenge after local or systemic vaccination of chickens with Newcastle disease virus. Infect Immun 1977; 18: $146-150$

17. Office International des Epizooties. Newcastle disease. In: Anonymous, Manual of standards for diagnostic tests and vaccines. 4th edn. Paris: Office International des Epizooties; 2000. p 221-232.

18. Collins MS, Bashiruddin JB, Alexander DJ. Deduced amino acid sequences at the fusion protein cleavage site of Newcastle disease viruses showing variation in antigenicity and pathogenicity. Arch Virol 1993; 128: 363-370.

19. Peeters BP, de Leeuw OS, Koch G, Gielkens AL. Rescue of Newcastle disease virus from cloned cDNA: evidence that cleavability of the fusion protein is a major determinant for virulence. J Virol 1999; 73: 5001-5009. 